\title{
Optical Solitons and Other Solutions to the (2+1)-Dimensional Cubic Nonlinear Schrödinger Equation with Fractional Temporal Evolution
}

\author{
Sibel Sehriban Atas $^{1}{ }^{*}$, , Tukur Abdulkadir Sulaiman ${ }^{1,2}$, and Hasan Bulut ${ }^{1,3}$ \\ ${ }^{1}$ Department of Mathematics, Firat University, Elazig, Turkey \\ ${ }^{2}$ Department of Mathematics, Federal University Dutse, Jigawa, Nigeria \\ ${ }^{3}$ Department of Mathematics Education, Final University, Girne, Cyprus
}

\begin{abstract}
In this study, the (2+1)-dimensional cubic nonlinear Schrödinger equation with fractional temporal evolution is investigated by using the extended sinh-Gordon equation expansion method. The idea of conformable fractional derivative is used in transforming the complex nonlinear partial differential equation to nonlinear ordinary differential equation. Dark, bright, mixed dark-bright, singular, mixed singular solitons and singular periodic wave solutions are successfully reached. The parametric conditions for the existence of valid solitons are given. The 2D and 3D graphics to some of the reported solutions are plotted.
\end{abstract}

\section{Introduction}

Nonlinear Shrodinger's type equations (NLSEs) are special kind of nonlinear evolution equations expressing several complex nonlinear aspect in the field of nonlinear sciences such as the optical fiber, fluid mechanics, plasma physics, biology, hydrodynamics and so on. Several analytical techniques have been used to find the solutions of various NLSEs [1-12].

In this study, the $(2+1)$-dimensional cubic nonlinear Schrödinger equation with fractional temporal evolution [13] is going to be soughted by using the extended sinh-Gordon equation expansion method (ShGEEM) [14-17].

The (2+1)-dimensional cubic nonlinear Schrödinger equation with fractional temporal evolution is given by [13]

$$
i D_{t}^{\alpha} \psi+c\left(\psi_{x x}+\psi_{y y}\right)+a|\psi|^{2} \psi+b \psi=0, \alpha \in(0,1),
$$

where $a, b, c$ are nonzero constant.

When $\alpha=1$, we have the original (2+1)-dimensional cubic nonlinear Schrödinger equation [13].

Corresponding author: sibel.s.atas@gmail.com 


\section{The Conformable Fractional derivative}

In this section, we give some basic definition, properties and theorem about the conformable fractional derivative.

Definition 1. Let $g:(0, \infty) \rightarrow \mathbb{R}$, then the conformable fraction derivative of $g$ of order $\alpha$, is defined as here, some basic properties of conformable fractional derivative are presented [18].

1. $T_{\alpha}(b g+c h)=b T_{\alpha}(g)+c T_{\alpha}(h), b, c \in \mathbb{R}$,

2. $T_{\alpha}\left(t^{\lambda}\right)=\lambda t^{\lambda-\alpha}, \lambda \in \mathbb{R}$,

3. $T_{\alpha}(g h)=g T_{\alpha}(h)+h T_{\alpha}(g)$,

4. $T_{\alpha}\left(\frac{g}{h}\right)=\frac{h T_{\alpha}(g)+g T_{\alpha}(h)}{h^{2}}$,

5. If $g$ is differentiable, then $T_{\alpha}(g)(t)=t^{1-\alpha} \frac{d g}{d t}$.

Theorem 1. Let $g, h:(0, \infty) \rightarrow \mathbb{R}$ be differentiable functions and also $\alpha$ differentiable, then the following rule holds:

$$
T_{\alpha}(g \circ h)(t)=t^{1-\alpha} h^{\prime}(t) g^{\prime}(h(t)) \text {. }
$$

\section{Application}

In this section, the application of the extended ShGEEM to the $(2+1)$-dimensional cubic nonlinear Schrödinger equation with fractional temporal evolution is presented.

Consider the complex time-fractional travelling wave transformation

$$
\psi(x, y, t)=\psi(\xi) e^{i \theta}, \theta=p x+q y+r \frac{t^{\alpha}}{\alpha}, \xi=\mu\left(x+y-k \frac{t^{\alpha}}{\alpha}\right)
$$

Inserting Eq. (3.1) into Eq. (1.1), we get the following NODE:

$$
\left(b-c\left(p^{2}+q^{2}\right)-r\right) \psi+a \psi^{3}+2 c \mu^{2} \psi^{\prime \prime}=0
$$

from the real part and the relation $k=2 c(p+q)$ from the imaginary part.

By the extended ShGEEM, the solutions of any given nonlinear partial differential equation are assumed to be of the forms [14]

$$
\psi(\theta)=\sum_{j=1}^{m}\left[b_{j} \sinh (\theta)+a_{j} \cosh (\theta)\right]^{j}+a_{0}
$$




$$
\psi(\xi)=\sum_{j=1}^{m}\left[ \pm i b_{j} \sec h(\xi)+a_{j} \tan h(\xi)\right]^{j}+a_{0}
$$

and

$$
\psi(\xi)=\sum_{j=1}^{m}\left[ \pm b_{j} \operatorname{csch}(\xi)+a_{j} \operatorname{coth}(\xi)\right]^{j}+a_{0}
$$

respectively.

Balancing the terms $\psi^{3}$ and $\psi^{\prime \prime}$ in Eq. (3.2), yields $m=1$.

With $m=1$, Eqs. (3.3)-(3.5) take the forms

$$
\begin{aligned}
& \psi(\theta)=b_{1} \sinh (\theta)+a_{1} \cosh (\theta)+a_{0} \\
& \psi(\xi)= \pm i b_{1} \sec h(\xi)+a_{1} \tan h(\xi)+a_{0}
\end{aligned}
$$

and

$$
\begin{aligned}
& \psi(\xi)= \pm b_{1} \operatorname{csch}(\xi)+a_{1} \cot h(\xi)+a_{0} \\
& \text { where } i=\sqrt{-1} \text { and } \theta^{\prime}=\sinh (\theta)[14]
\end{aligned}
$$

Inserting Eq. (3.6) and its second derivative into Eq. (3.2) gives a polynomial in powers of hyperbolic functions. Summing each coefficient of the hyperbolic functions of the same power and equating each summation to zero, gives a group of algebraic equations. The system of algebraic equations is simplified to secure the values of the parameters involved. For each case, inserting the obtained values of the parameters into each of Eqs. (3.6) and (3.8), yields the solutions to Eq. (1.1). Simplifying the group of algebraic equation, we get the values of the parameters involved.

Case 1: When

$$
a_{0}=0, a_{1}=-\frac{2 \sqrt{-c} \mu}{\sqrt{a}}, b_{1}=0, q=\frac{\sqrt{b-r-c\left(q^{2}+4 \mu^{2}\right)}}{\sqrt{c}},
$$

we have the following dark and singular

soliton: $u_{1}(x, t)=-\frac{2 \sqrt{-c} \mu\left(\tanh \left[\left(x+y-\frac{k t^{\alpha}}{\alpha}\right) \mu\right]\right)}{\sqrt{a}} \mathrm{e}^{i\left(p x+q y+r \frac{t^{\alpha}}{\alpha}\right)}$ 
$u_{2}(x, t)=-\frac{2 \sqrt{-c} \mu\left(\operatorname{coth}\left[\left(x+y-\frac{k t^{\alpha}}{\alpha}\right) \mu\right]\right)}{\sqrt{a}} \mathrm{e}^{i\left(p x+q y+r \frac{t^{\alpha}}{\alpha}\right)}$,

where $a c<0$ and $c\left(b-r-c\left(q^{2}+\mu^{2}\right)\right)>0 \quad$ for valid soliton.

Case 2: When

$$
a_{0}=0, a_{1}=0, b_{1}=\frac{2 \sqrt{-c} \mu}{\sqrt{a}}, q=-\frac{\sqrt{b-r-c\left(q^{2}-2 \mu^{2}\right)}}{\sqrt{c}},
$$

we have the following bright and singular

soliton: $u_{3}(x, t)=-\frac{2 \sqrt{-c} \mu\left(\operatorname{sech}\left[\left(x+y-\frac{k t^{\alpha}}{\alpha}\right) \mu\right]\right)}{\sqrt{a}} \mathrm{e}^{i\left(p x+q y+r+\frac{t^{\alpha}}{\alpha}\right)}$,

where $c\left(b-r-c\left(q^{2}+\mu^{2}\right)\right)>0 \quad$ for valid soliton.

$u_{4}(x, t)=\frac{2 i \sqrt{c} \mu\left(\operatorname{csch}\left[\left(x+y-\frac{k t^{\alpha}}{\alpha}\right) \mu\right]\right)}{\sqrt{a}} \mathrm{e}^{i\left(p x+q y+r \frac{r^{\alpha}}{\alpha}\right)}$,

where $c\left(b-r-c\left(q^{2}+\mu^{2}\right)\right)>0 \quad$ for valid soliton.
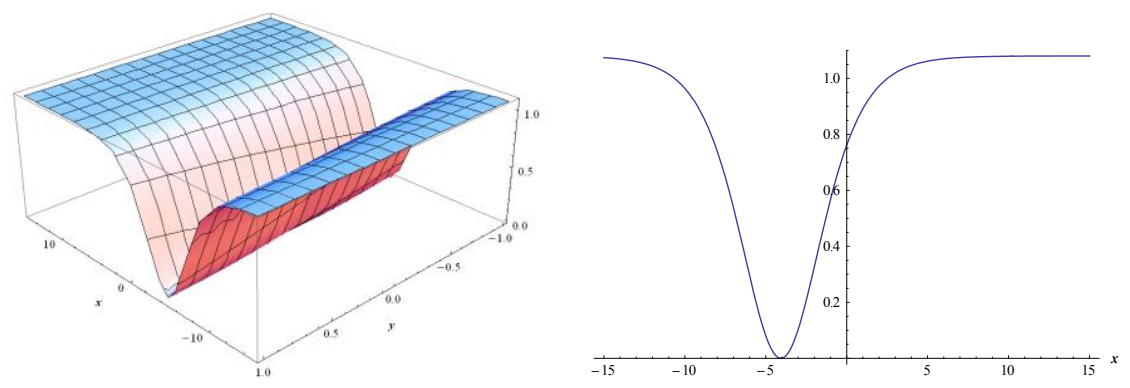

Figure 1. The $3 \mathrm{D}$ and $2 \mathrm{D}$ surfaces of Eq. (3.10) under the values of $c=-3, a=1, k=-0.75, \mu=0.3, \alpha=0.2,-15<x<15,-1<y<1$ and $t=0.5$ for the 2D surface. 

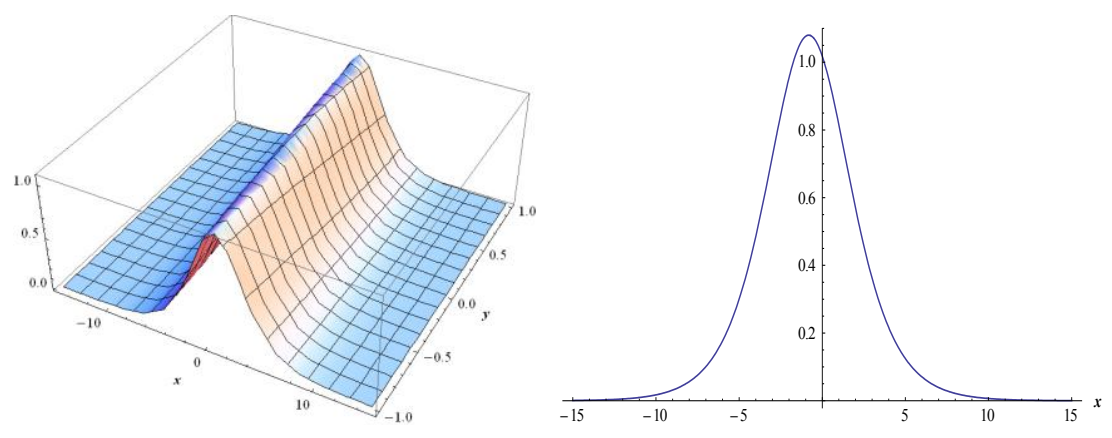

Figure 2. The $3 \mathrm{D}$ and $2 \mathrm{D}$ surfaces of Eq. (3.13) under the values of $c=-3, a=1, k=-0.003, \mu=0.3, \alpha=0.2, y=0.8,-15<x<15,-1<y<1$ and $t=0.5$ for the 2D surface.

\section{Conclusion}

In this work, the extended sinh-Gordon equation expansion method is used in extracting various soliton and other solutions such as the dark, bright and singular solutions to the $(2+1)$ dimensional cubic nonlinear Schrödinger equation with fractional temporal evolution. Using suitable values of parameters, the 2- and 3-dimensional surfaces to some of the reported solutions are plotted. The reported solutions in this study may be useful in explaining the physical meaning of several nonlinear models arising in the different fields of nonlinear sciences. The extended ShGEEM provides a powerful mathematical tool for securing family of wave solutions to different kind of nonlinear models.

\section{References}

1. H. Bulut, T.A. Sulaiman, B. Demirdag, Nonlinear Dyn 91(3), 1985-1991 (2018)

2. S. Duran, M. Askin, T.A. Sulaiman, IJOCTA 7(3), 240-247 (2017)

3. H. Bulut, H.A. Isik, T.A. Sulaiman, ITM Web of Conferences 13, 01019 (2017)

4. H. Bhrawy, M. A. Abdelkawy, A. Biswas. Indian J. Phys 87,1125-1131 (2013)

5. H.M. Baskonus, H. Bulut, Waves in Rnd. and Comp. Med 26, 189-196 (2016)

6. F. Özpinar, H. M. Baskonus and H. Bulut, Entropy 17(12), 8267-8277 (2015)

7. H.M. Baskonus, H. Bulut, Waves in Rnd. and Comp. Med 25(4), 720-728 (2015)

8. F. Belgacem, A.A. Karaballi, and S.L. Kalla, Math. Prob. Eng. 3-4,103 (2003)

9. A. Yokus, H.M. Baskonus, T.A. Sulaiman and H. Bulut, Numer Methods Partial Differential Eq. 34, 211-227 (2018)

10. A. Yokus, T.A. Sulaiman and H. Bulut, Opt Quant Electron 50, 31 (2017)

11. H. Bulut, T.A. Sulaiman, H.M. Baskonus and A.A. Sandulyak, Optik 135, 327336 (2017)

12. H. Bulut, T.A. Sulaiman, H.M. Baskonus and T. Akturk, Opt Quant Electron 50, 134 (2018) 
13. N. Taghizadeh, M. Mirzazadeh, , International Journal of Applied Mathematics and Computation, 5(3) 12-16 (2013)

14. H.M. Baskonus, T.A. Sulaiman, H. Bulut, T. Akturk, Superlattices Microstruct, 115 19-29 (2018)

15. C. Cattani, T.A. Sulaiman, H.M. Baskonus and H. Bulut, Opt Quant Electron 50 $138,(2018)$

16. C. Cattani, T.A. Sulaiman, H.M. Baskonus and H. Bulut, Eur. Phys. J. Plus 133, 228 (2018)

17. H.M. Baskonus, T.A. Sulaiman and H. Bulut, Opt Quant Electron 50, 253 (2018)

18. R. Khalil, M. Al Horani, A. Yousef, M. Sababheh, Journal of Computational and Applied Mathematics, 264 65-70 (2014) 